pháp an toàn, hiệu quả, tiết kiệm chi phí, sớm phục hồi chức năng chi cho người bệnh.

\section{TÀI LIẸU THAM KHẢO}

1. Johansen $S$. Outcome after knee dislocations: a 2-9 years follow-up of 85 consecutive patients. Knee Surg Sports Traumatol Arthrosc. 2009;17(9):1013-26.

2. Klimkiewicz JJ, Petrie RS, Harner CD. Surgical treatment of com- bined injury to anterior cruciate ligament, posterior cruciate liga- ment, and medial structures. Clin Sports Med. 2000;19(3):479-92.

3. Liow RY, McNicholas MJ, Keating JF, Nutton RW. Ligament repair and reconstruction in traumatic dislocation of the knee. J Bone Joint Surg Br. 2003;85(6):845-51.

4. Wong CH, Tan JL, Chang HC, Khin LW, Low Co. Knee dislocations-a retrospective study comparing operative versus closed immobi- lization treatment outcomes. Knee Surg Sports Traumatol Arthrosc. 2004;12(6):540

5. Wascher DC, Becker JR, Dexter JG, Blevins FT. Reconstruction of the anterior and posterior cruciate ligaments after knee disloca- tion. Results using fresh-frozen nonirradiated allografts. Am J Sports Med. 1999;27(2):189-96.

6. Mariani PP, Margheritini F, Camillieri G. Onestage arthroscopi- cally assisted anterior and posterior cruciate ligament recon- struction. Arthroscopy. 2001;17(7):700-7. U, Wippermann B, Hofmann A, K

7. Fanelli GC, Edson Cj. Conbined $\mathrm{PCL}$ posterolateral reconstructions with Achilles tendon allograft and biceps femoris tendon tenodesis: 2to 10-year follow-up. Arthros- copy. 2004; 20(4): $339-45$.

\title{
MộT Số YẾU Tố LIÊN QUAN ĐẾN CHẤP NHÂ̂N TIÊM VẮC XIN PHÒNG COVID-19 CỦA GIÁO VIÊN VIÊTT NAM NĂM 2021
}

\section{TÓM TẮT}

Nghiên cứu mô tả cắt ngang trên 1902 giáo viên ở Việt Nam có độ tuổi từ 18- 59 từ ngày 15/5/2021 đến $16 / 6 / 2021$. Mục tiêu nghiên cứu nhằm xác định tỷ lệ và môt số yếu tố liên quan đến chấp nhân tiêm vắc xin phòng COVID-19 của giáo viên Việt Nam. Kết quả: tỷ lệ đối tượng nghiên cứu chấp nhân tiêm vắc xin là $85,9 \% ; 69,1 \%$ săn sàng trả tiền tiểm vắc xin. Các yếu tố liên quan đến tỷ lệ chấp nhân tiêm vắc xin là nhóm tuổi từ 40 đến 49 tuổi và từ 50 đến 59 tuổi sẵn sàng tiêm vắc xin gấp 2,05 lần và 2,67 lần so với nhóm từ $18-29$ tuổi $(p<0,001)$, giáo viên cấp THCS và THPT tỷ lệ chấp nhận tiêm ít hơn so với nhóm giáo viên mầm non $(\mathrm{p}<0,001)$, nhóm giáo viên có gia đình sẵn sàng tiêm vắc xin gấp 2,21 lần so với nhóm còn độc thân $(p<0,001)$, tình trạng mắc bệnh mạn tính làm giảm tỷ lệ chấp nhận tiêm vắc xin $(p<0,001)$; chưa tìm thây sư khác biêt về viêc chấp nhân tiêm phòng vắc xin COVID-19 giữa thành phố và nông thôn, nhóm nam và nữ, giữa người dân tộc Kinh với những người dân tộc khác. Kết luận: kết quả nghiên cứu cho thây tỷ lệ giáo viên chấp nhận tiêm vắc xin cao, đa số châp nhận chi trả cho việc tiêm vắc xin. Các yếu tố liên quan đến tỷ lệ chấp nhận tiêm vắc xin có ý nghĩa là nhóm tuổi, cấp giảng dạy, tình trạng hôn nhân và tình trạng mắc bệnh mạn tính.

\footnotetext{
${ }^{1}$ Trường Đại học Y tế Công cộng

${ }^{2}$ Trường Đại học Kinh tế Quốc dân

Chiu trách nhiệm chính: Nguyễn Thị Hà

Email: nth16@huph.edu.vn

Ngày nhận bài: 14.5.2021

Ngày phản biên khoa họ: 1.7.2021

Ngày duyệt bài: 12.7.2021
}

Nguyễn Thị Hà ${ }^{1}$ Đặng Thị Huê², Phạm Hùng Tiến', Nguyễn Thị Anh Vân ${ }^{1}$

Từ khóa: Vắc xin phòng COVID-19; chấp nhận tiêm vắc xin; giáo viên Việt Nam.

\section{SUMMARY \\ SOME FACTORS ASSOCIATED WITH \\ ACCEPTANCE OF COVID-19 VACCINE \\ AMONG VIETNAMESE TEACHERS}

A cross-sectional web-based study was conducted from May 15, 2021 to June 16, 2021. The study participants included 1902 Vietnamese teachers, between 18 and 59 years old. The objective of the study was to determine the rate and some factors associated with acceptance of COVID-19 vaccine among Vietnamese teachers. Results: $85.9 \%$ of the participants accepted vaccination; $69.1 \%$ confirmed their willingness to pay for vaccination. Some factors as age, being a secondary school teacher, marital status, post-graduate education, and chronic diseases are the related factors to their vaccination acceptance; no difference was found between city and countryside, between male and female teachers, between Kinh and other ethnic groups in terms of their acceptance of COVID-19 vaccination.

Keywords: COVID-19 vaccine; vaccination acceptance; Vietnamese teachers.

\section{I. ĐĂT VẤN ĐỀ}

Dich bênh COVID-19 lần đầu tiên được báo cáo ở Vũ Hán-Trung Quốc vào ngày 31 tháng 12 năm 2019 [1]. Cho đến nay, dịch COVID-19 đã lan ra toàn cầu với số lượng người tử vong rất cao. Thống kê đến ngày $21 / 7 / 2021$, trên thế giới có 188.655.968 người mắc COVID-19 với tổng số ca tử vong là 4.067.517 [2]. Tại Việt Nam, vào 
tháng 6/2021, đang trong làn sóng thứ 4 của dịch COVID-19, diễn biến dịch bệnh phức tạp và đang lan nhanh ra nhiều tỉnh thành trong cả nước. Theo thống kê từ Bộ Y tế đến ngày 19/7 cả nước có 43.458 ca nhiễm và 254 trường hợp đã tử vong [2].

Cũng giống như các bệnh truyền nhiễm khác, bên cạnh các biện pháp phòng chống sự lây lan của dịch dựa trên nguyên tắc cắt đứt nguồn lây nói chung, tiêm vắc xin là biện phát phòng bệnh có tính quyết định. Ngay từ cuối năm 2020, vắc xin phòng COVID-19 đã được tiêm cho người dân tại một số nước như Trung Quốc, Mỹ, tiếp đó lần lượt các quốc gia khác như Anh, Cộng hòa liên bang Nga, Ấn Độ,... Tại Việt Nam, vắc xin COVID-19 bắt đầu được tiêm vào ngày 8 tháng 3 năm 2021, cho đến ngày 21 tháng 7 năm 2021 chỉ có $13,2 \%$ dân số được tiêm đủ liều vắc xin [2]. Một số nghiên cứu trên thế giới cho thấy tỷ lệ người dân chấp nhận tiêm vắc xin phòng COVID-19 còn thấp. Một nghiên cứu trên 3.100 người tại Jordan (2020) cho thây chỉ có $37,4 \%$ sẳn sàng chấp nhận tiêm vắc xin [3]. Tỷ lệ này trong nghiên cứu tại Kuwiat là $53,1 \%$ [5]; và tại Trung Đông là 36,8\% [6]. Thực tế tại một số nước đã triển khai tiêm chủng rộng rãi, khi tỷ lệ tiêm chủng chưa đạt miễn dịch cộng đồng, tốc độ tiêm chủng đã bắt đầu chậm lại do nhiều người không muốn tiêm vắc xin.

Tại Việt Nam chưa có nghiên cứu nào về chấp nhận tiêm vắc xin đối với giáo viên, những người thường xuyên tiếp xúc với nhiều học sinh, nếu giáo viên bị mắc bệnh sẽ có khả nang lây bệnh cho nhiều người. Nghiên cứu này nhằm xác định tỷ lệ chấp nhận tiêm vắc xin phòng COVID-19 và một số yếu tố liên quan ở giáo viên Việt Nam. Kết quả của nghiên cứu cung cấp những bằng chứng khoa học, có thể sử dụng trong truyền thông, lập và thực hiện thành công kế hoạch tiêm chủng vắc xin phòng COVID-19 tại Việt Nam.

\section{II. ĐỐI TƯợNG VÀ PHƯƠ'NG PHÁP NGHIÊN CỨU}

2.1. Đối tượng nghiên cứu: Đối tượng nghiên cứu là giáo viên các trường từ mầm non đến trung học phổ thông (THPT), tuổi từ 18 đến 59, đang sinh sống tại VN trong thời gian thu thập số liệu.

2.2. Phương pháp nghiên cứu: Thiết kế nghiên cứu mô tả cắt ngang.

Cõ̃ mẫu và phương pháp chọn mẫu: phương pháp chọn mẫu thuận tiện được áp dụng trong nghiên cứu này. Thư mời tham gia nghiên cứu và bộ câu hỏi được gửi qua mạng xã hội (zalo, viber, facbook,...) tới các GV mà nhóm nghiên cứu quen biết; những GV này chuyển tiếp thư mời và bộ câu hỏi tới những GV trong mạng xã hội của họ và từ đó tiếp tục tới những GV khác. Sau khi đọc thư mời, nếu GV chấp nhận tự nguyện tham gia nghiên cứu, sẽ bắt đầu trả lời câu hỏi.

Phướng pháp thu thập số liệu: đối tương nghiên cứu đồng ý nghiên cứu trả lời câu hỏi trực tiếp trên mạng xã hội. Thời gian thu thập số liệu từ 15 đến 25 tháng 5 năm 2021. Tổng số 1902 giáo viên đã tham gia nghiên cứu và hoàn thành bộ câu hỏi.

Chỉ tiêu nghiên cứu chính bao gồm:

- Đặc điểm nhân khẩu học

- Thông tin tìm hiểu về vắc xin phòng COVID-19.

- Quan điểm đối với vắc xin phòng COVID-19.

- Sự chấp nhận tiêm vắc xin phòng COVID-19 và mối liên quan giữa một số đặc điểm nhân khẩu học với việc chấp nhận tiêm vắc xin phòng COVID-19.

2.3. Phương pháp phân tích số liệu: số liệu được phân tích bằng phần mềm Stata 14.0. Các phép tính thống kê thông thường được sử dụng để tính tần suất, tỷ lệ các biến số nghiên cứu. Phân tích sử dụng kiểm định $X^{2}$, tỉ suất chênh (OR) và khoảng tin cậy 95\% (KTC 95\%) được tính để xác định mối liên quan giữa một số biến nhân khẩu học của đối tượng nghiên cứu với biến đầu ra "chấp nhận tiêm vắc xin COVID-19".

2.4. Vấn đề đạo đức nghiên cứu: nghiên cứu được thông qua Hội đồng đạo đức trong NCYSH Trường ĐH Y tế Công cộng (Quyết định số 204/2021/YTCC-HD3, ngày 6/5/ 2021). Những quy định về đạo đức trong nghiên cứu đã được thực hiện nghiêm túc trong suốt quá trình nghiên cứu.

\section{KẾT QUẢ NGHIÊN CỨU}

3.1. Một số đặc điểm nhân khẩu học của đối tượng nghiên cứu

Bảng 6. Đặc điểm nhân khẩu học của đôi tượng nghiên cứu

\begin{tabular}{|c|c|c|c|}
\hline \multicolumn{2}{|c|}{ Đặc điểm (N=1902) } & $\begin{array}{c}\text { Tống } \\
\text { số (n) }\end{array}$ & $\begin{array}{c}\text { Tỉ lề } \\
\mathbf{( \% )}\end{array}$ \\
\hline \multirow{2}{*}{ Nơi sống } & Thành phố & 1491 & 78,4 \\
\cline { 2 - 4 } & Nông thôn & 411 & 21,6 \\
\hline \multirow{3}{*}{$\begin{array}{c}\text { Nhóm } \\
\text { tuổi }\end{array}$} & $18-29$ & 203 & 10,7 \\
\cline { 2 - 4 } & $30-39$ & 483 & 25,4 \\
\cline { 2 - 4 } & $40-49$ & 865 & 45,5 \\
\cline { 2 - 4 } & $50-59$ & 351 & 18,4 \\
\hline \multirow{4}{*}{ Trường } & Mầm non & 546 & 29,2 \\
\cline { 2 - 4 } & Tiếu học & 790 & 42,3 \\
\cline { 2 - 4 } & THCS & 393 & 21,0 \\
\cline { 2 - 4 } & THPT & 140 & 7,5 \\
\hline
\end{tabular}




\begin{tabular}{|c|c|c|c|}
\hline \multirow{2}{*}{ Giới tính } & Nam & 162 & 8,5 \\
\hline & Nữ & 1740 & 91,5 \\
\hline \multirow{3}{*}{$\begin{array}{c}\text { Tình } \\
\text { trạng hôn } \\
\text { nhân }\end{array}$} & $\begin{array}{c}\text { Chưa lập gia } \\
\text { định }\end{array}$ & 109 & 5,7 \\
\hline & $\begin{array}{c}\text { Đang có } \\
\text { vợ/chồng }\end{array}$ & 1658 & 87,2 \\
\hline & $\begin{array}{l}\text { Ly hôn/ly } \\
\text { thân/Góa }\end{array}$ & 135 & 7,1 \\
\hline \multirow{2}{*}{ Dân tộc } & Kinh & 1767 & 92,9 \\
\hline & Khác & 135 & 7,1 \\
\hline \multirow{3}{*}{$\begin{array}{l}\text { Trình đô } \\
\text { học vấn } \\
\text { cao nhất }\end{array}$} & Trung cấp & 130 & 6,8 \\
\hline & $\begin{array}{c}\text { Cao đắng/Đại } \\
\text { hoc }\end{array}$ & 1657 & 87,1 \\
\hline & Sau Đại học & 115 & 6,1 \\
\hline \multirow{2}{*}{$\begin{array}{l}\text { Mắc bệnh } \\
\text { mạn tính }\end{array}$} & Không & 1640 & 86,2 \\
\hline & Có & 262 & 13,8 \\
\hline
\end{tabular}

\begin{tabular}{|c|c|c|c|}
\hline \multirow{2}{*}{$\begin{array}{c}\text { Tiền sử dị } \\
\text { ứng }\end{array}$} & Không & 1119 & 58,8 \\
\cline { 2 - 4 } & Có & 252 & 13,3 \\
\cline { 2 - 4 } & Không biết & 531 & 27,9 \\
\hline
\end{tabular}

Kết quả bảng 1 cho thây trong tổng số 1902 đối tượng nghiên cứu (ĐTNC), nữ chiếm 91,5\%; nhóm tuổi 18-29 chiếm tỷ lệ thấp nhất $(10,7 \%)$, nhóm 40-49 tuổi chiếm tỉ lệ cao nhất $(45,5 \%)$; ĐTNC ở thành phố chiếm tỉ lệ $78,4 \%$ và ở nông thôn là 21,6\%; tỷ lệ ĐTNC là giáo viên tiểu học, mầm non, THCS và THPT chiếm tỷ lệ tương ứng $42,3 \% ; 29,2 \% ; 21,0 \%$ và $7,5 \%$; ĐTNC là dân tộc kinh chiếm $92,9 \%$. Về tình trang hôn nhân, ĐTNC đang có vợ/chồng chiếm $87,2 \%$, ly hôn/ly thân/góa chiếm $7,1 \%$, còn lại là chưa lập gia đình; tỉ lệ mắc bệnh mãn tính là $13,8 \%$; số người có tiền sử dị ứng chiếm 13,3\%.

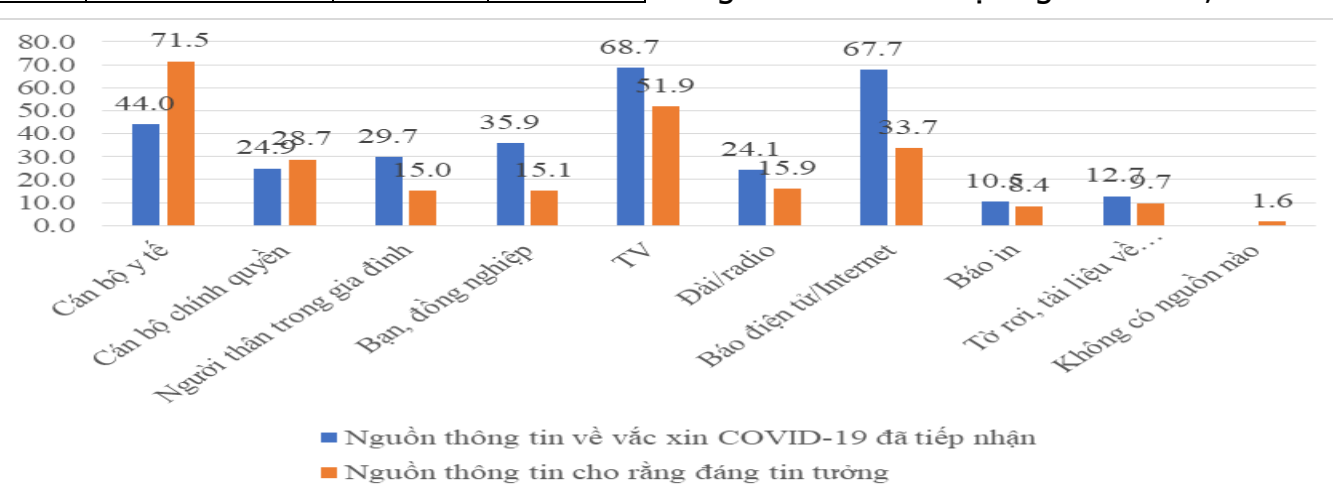

Biểu đồ 1. Nguồn thông tin và sự tin tưởing vào nguồn thông tín về vắc xín COVID-19

Biểu đồ 1 cho thấy, có ba nguồn thông tin về vắc xin được ĐTNC tiếp cận nhiều nhất là truyền hình $(68,7 \%)$, báo điện tử/internet $(67,7 \%)$ và cán bộ y tế là $44,0 \%$. Nguồn thông tin tin cậy nhất là từ cán bộ y tế $(71,5 \%)$, tiếp đến là truyền hình $(51,9 \%)$ và báo điện tử/internet là $33,7 \%$.

\subsection{Quan điểm của đối tượng nghiên cứu đối với vắc xin phòng COVID-19}

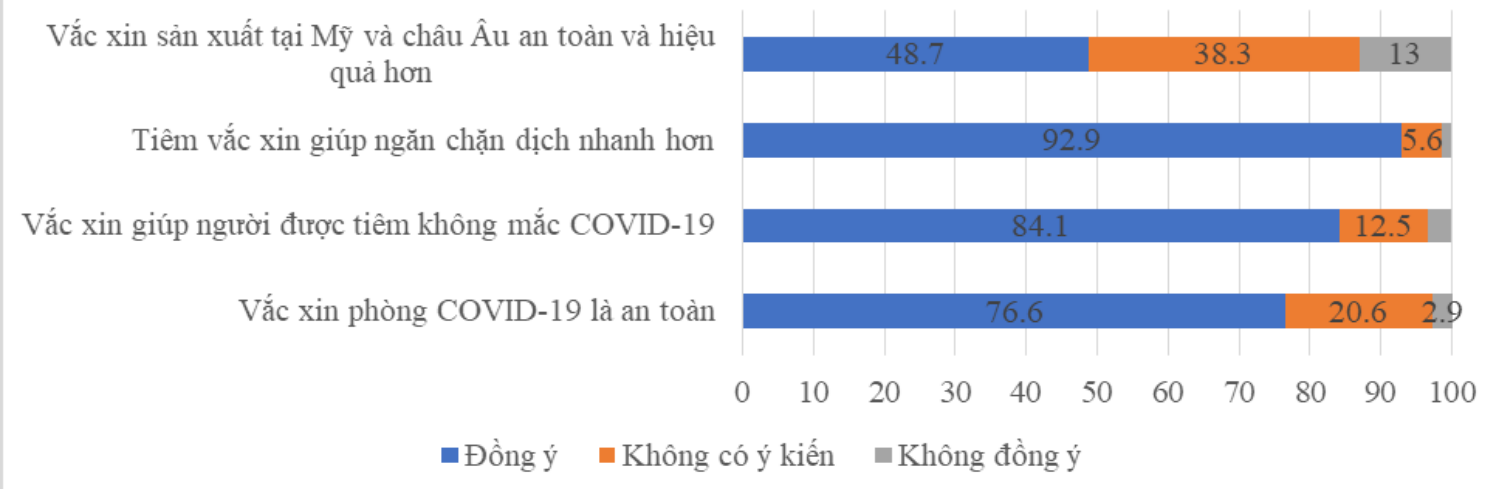

\section{Biểu đồ 2. Quan điểm của đôii tượng nghiên cứu về vắc xin phòng COVID-19}

Biểu đồ 2 cho thây $92,9 \%$ ĐTNC đồng ý với nhận định tiêm vắc xin giúp ngăn chặn dịch bệnh nhanh hơn (92,9\%). Tỉ lệ đồng ý với nhận định vắc xin giúp người tiêm không mắc COVID-19 chiếm tỉ lệ lớn nhất $(84,1 \%)$, không có ý kiến $(12,5 \%)$ và không đồng ý $(3,4 \%)$. 76,6\% ĐTNC cho rằng vắc xin là an toàn, số không có ý kiến chiếm $20,6 \%$ và $2,9 \%$ cho rằng vắc xin không an toàn. Với nhận định "vắc xin sản xuất tại Mỹ và châu Âu là an toàn và hiệu quả hơn vắc xin sản xuất ở nơi khác" thì tỉ lệ đồng ý, không đồng ý, không có ý kiến chiếm tỉ lệ lần lượt là 48,7\% ; 
$38,3 \%$ và $13,0 \%$.

3.3. Quyết định tiêm vắc xin và một số yếu tố liên quan đến quyết định tiêm vắc xin của đối tượng nghiên cứu

Bảng 2. Một số đặc điểm về quyêt định tiêm phòng COVID-19 của đôi tượng nghiên cứu

\begin{tabular}{|c|c|c|c|}
\hline \multicolumn{2}{|c|}{ Đặc điểm (N=1902) } & $\begin{array}{c}\text { Tống số } \\
\text { (n) }\end{array}$ & $\begin{array}{c}\text { Tỷ lệ } \\
(\mathbf{\%})\end{array}$ \\
\hline \multirow{2}{*}{$\begin{array}{c}\text { Sẵn sàng } \\
\text { tiêm }\end{array}$} & Có & 1634 & 85,9 \\
\cline { 2 - 4 } & Không & 15 & 0,8 \\
\cline { 2 - 4 } & $\begin{array}{c}\text { Chưa quyết } \\
\text { định }\end{array}$ & 253 & 13,3 \\
\hline
\end{tabular}

\begin{tabular}{|c|c|c|c|}
\hline \multirow{2}{*}{$\begin{array}{c}\text { Sã̃n sàng trả } \\
\text { tiền cho việc } \\
\text { tiêm }\end{array}$} & Có & 1315 & 69,1 \\
\cline { 2 - 4 } & $\begin{array}{c}\text { Không } \\
\text { Chưa quyết } \\
\text { định }\end{array}$ & 103 & 5,4 \\
\hline $\begin{array}{c}\text { Sằn sàng } \\
\text { tiêm vắc xin } \\
\text { do VN sản } \\
\text { xuất }\end{array}$ & Có & 1401 & 25,5 \\
\cline { 2 - 4 } & Không biết & 43,7 \\
\cline { 2 - 4 } & Không & 61 & 3,2 \\
\hline
\end{tabular}

Bảng 2 cho thấy 85,96\% ĐTNC chấp nhân tiêm vắc xin và $69,1 \%$ trả lời sẵn sàng chi trả; ĐTNC sẵn sàng tiêm vắc xin do VN sản xuất chiếm tỉ lệ lớn nhất $(73,7 \%) ; 3,2 \%$ không tiêm và $23,1 \%$ trả lời là không biết.

Bảng 3. Môi liên quan giữa đặc điểm cá nhân của đôi tượng nghiên cứu với việc sã̃n sàng tiêm vắc xin phòng COVID-19

\begin{tabular}{|c|c|c|c|c|}
\hline \multirow{2}{*}{ Đặc điểm $(N=1902)$} & \multicolumn{2}{|c|}{$\begin{array}{l}\text { Săn sàng tiêm vắc xin phòng } \\
\text { COVID-19 }\end{array}$} & \multirow{2}{*}{$\begin{array}{c}\text { OR } \\
\text { (KTC 95\%) }\end{array}$} & \multirow{2}{*}{$\mathbf{P}$} \\
\hline & $\begin{array}{c}\text { Có } \\
\text { N (\%) }\end{array}$ & $\begin{array}{c}\text { Không/ Chưa } \\
\text { quyết định } \mathbf{N}(\%)\end{array}$ & & \\
\hline Nơi sống: Thành phố & $1290(86,5)$ & $201(13,5)$ & \multirow{2}{*}{$0,8(0,59-1,08)$} & \\
\hline Nông thôn & $344(83,7)$ & $67(16,3)$ & & $>0,05$ \\
\hline Nhóm tuối: 18 - 29 & $159(78,3)$ & $44(21,7)$ & 1 & \\
\hline $30-39$ & $395(81,8)$ & $88(18,2)$ & $1,24(0,83-1,86)$ & $>0,05$ \\
\hline $40-49$ & $762(88,1)$ & $103(11,9)$ & $2,05(1,38-3,03)$ & $<0,001$ \\
\hline $50-59$ & $318(90,6)$ & $33(9,4)$ & $2,67(1,63-4,35)$ & $<0,001$ \\
\hline Giới tính: Nam & $137(84,6)$ & $25(15,4)$ & \multirow{2}{*}{$1,12(0,72-1,76)$} & \\
\hline$N \tilde{u}$ & $1497(86,0)$ & $243(14,0)$ & & $>0,05$ \\
\hline Giáo viên cấp: Mầm non & $484(88,6)$ & $62(11,4)$ & 1 & \\
\hline Tiếu học & $710(89,9)$ & $80(10,1)$ & $1,14(0,80-1,62)$ & $>0,05$ \\
\hline THCS & $313(79,6)$ & $80(20,4)$ & $0,50(0,35-0,72)$ & $<0,001$ \\
\hline THPT & $106(75,7)$ & $34(24,3)$ & $0,40(0,25-0,64)$ & $<0,001$ \\
\hline \multicolumn{5}{|l|}{ Tình trạng hôn nhân } \\
\hline Chưa lập gia đình & $81(74,3)$ & $28(25,7)$ & 1 & \\
\hline Đang có vợ/chồng & $1434(86,5)$ & $224(13,5)$ & $2,21(1,41-3,48)$ & $<0,001$ \\
\hline Ly hôn/ly thân/Góa & $119(88,2)$ & $16(11,8)$ & $2,57(1,31-5,05)$ & $<0,05$ \\
\hline Dân tộc: Kinh & $1514(85,7)$ & $253(14,3)$ & \multirow{2}{*}{$1,34(0,77-2,32)$} & \\
\hline Khác & $120(88,9)$ & $15(11,1)$ & & $>0,05$ \\
\hline \multicolumn{5}{|l|}{ Trình độ học vấn cao nhất } \\
\hline Trung cấp & $115(88,5)$ & $15(11,5)$ & 1 & \\
\hline Cao đằng/Đại học & $1431(86,4)$ & $226(13,6)$ & $0,83(0,48-1,44)$ & $>0,05$ \\
\hline Sau Đại học & $88(76,5)$ & $27(23,5)$ & $0,43(0,21-0,85)$ & $<0,05$ \\
\hline \multicolumn{5}{|l|}{ Mắc bệnh mạn tính } \\
\hline Không & $1433(87,4)$ & $207(12,6)$ & \multirow{2}{*}{$0,48(0,35-0,66)$} & \\
\hline Có & $201(76,7)$ & $61(23,3)$ & & $<0,001$ \\
\hline
\end{tabular}

Bảng 3 cho thấy tỷ lệ chấp nhận tiên vắc xin của nhóm ĐTNC thuộc nhóm tuổi 40-49 và 50-59 cao hơn gấp 2,05 và 2,67 lần so với nhóm 18-29 tuổi $(p<0,001)$. Giáo viên cấp THCS và THPT chấp nhận tiêm vắc xin ít hơn GV cấp mần non 0,5 và 0,4 lần $(p<0,001)$; những người đang có vợ/chồng cao hơn so với những người chưa lập gia đình 2,21 lần $(p<0,001)$; tỉ lệ chấp nhận tiêm vắc xin ở nhóm người có trình độ học vấn sau
ĐH thấp hơn so với những người có trình độ học vấn là trung cấp 0,43 lần $(p<0.05)$; những người mắc bệnh mạn tính chấp nhận tiêm vắc xin thấp hơn 0,48 lần so với những người không mắc bệnh $(p<0.001)$.

\section{BÀN LUẬN}

4.1. Một số đặc điểm của giáo viên tham gia khảo sát về vắc xin phòng COVID-19. Trong tổng số 1902 ĐTNC có 78,4\% đến từ 
thành phố và chủ yếu trong đô tuổi từ 40 đến 49 tuổi. Tỷ lệ đang có vơ/chồng là $74,2 \%$ và do đặc thù nghề nghiệp là giáo viên nên tỷ lệ nữ trong nghiên cứu của chúng tôi chiếm đa số $(91,5 \%)$ và $13,8 \%$ là đang mắc bênh man tính. Tìm hiểu về sự quan tâm đối với vắc xin COVID-19 có $98,6 \%$, tỷ lệ này tại Kuwait trong nghiên cứu trên 2368 người trưởng thành là $71,2 \%$ [4]. Thông tin về vắc xin phòng COVID-19 được tiếp cận từ nhiều nguồn, trong đó cao nhất là từ ti vi 68,7\%, nhưng thông tin được tin cậy nhất từ lại từ cán bộ y tế $(71,5 \%)$, điêu này phù hợp với nghiên cứu tại Hoa kỳ (Nguồn thông tin tin cậy nhất từ các chuyên gia chăm sóc sức khỏe $(75 \%))[6]$

4.2. Quan điểm đôi với vắc xin phòng COVID-19. Tỉ lệ ĐTNC đồng ý với việc tin rằng vắc xin phòng COVID-19 là an toàn chiếm $76,6 \%$, tỷ lệ này tương đồng với nghiên cứu trên 2528 người trưởng thành tại Malaysia $(79,8 \%)$ [8]. $84,1 \%$ người được khảo sát tin rằng tiêm vắc xin phòng COVID-19 sẽ giúp người tiêm tránh mắc bệnh COVID-19 và $92,9 \%$ ngăn chặn dịch COVID-19 nhanh hơn, quan điểm này khá tương đồng với khảo sát taii Trung Quốc (89,5\% cho rằng tiêm chủng là cách hiệu quả để ngăn ngừa và kiểm soát COVID-19) [7]. Có gần một nửa $(48,7 \%)$ số người khảo sát cảm thấy vắc xin sản xuất tại Mỹ và châu Âu an toàn và hiệu quả hơn.

4.3. Quyết định tiêm vắc xin phòng phòng COVID-19 và mối liên quan giữa nhân khẩu học với việc sẵn sàng tiêm vắc xin phòng phòng COVID-19. Tỉ lệ ĐTNC sẵn sàng tiêm vắc xin là $85,9 \%$, tỉ lệ này cao so với các nước trên thế giới $(53,1 \%$ ở Kuwait [4], $36,8 \%$ ở Trung Đông [5], 67\% ở Hoa kỳ [6], 91,3\% ở Trung Quốc [7]). Bên cạnh đó tî lế ĐTNC sẵn sàng trả tiền cho việc tiêm vắc xin phòng COVID-19 $(69,1 \%)$ cũng chiếm tỉ lệ khá lớn. Vắc xin do VN sản xuất cũng được ĐTNC tin tưởng và lựa chọn $(73,7 \%)$.

Tìm hiểu về những yễu tố liên quan đến quyết định chấp nhận tiêm vắc xin chúng tôi thấy có đặc điểm về độ tuổi từ 40-49 và từ 50 59 cao hơn gấp 2,05 lần [KTC95\%: 1,38 - 3,03] và 2,67 lần [KTC95\%: 1,63 - 4,35] so với nhóm tuổi $18-29(p<0,001)$; kết quả này trái ngược với kết quả của nghiên cứu tại Mỹ (nhóm tuổi từ 18 đến 29 tuổi $(71 \%)$ nhiều hơn 50 đến 64 tuổi (64\%)) [6]. Điều này cũng có thể giải thích bởi sự khác nhau giữa các quốc gia và nhận thức về Vắc xin giữa các nhóm tuổi. Giáo viên cấp THCS và THPT chấp nhân tiêm thấp hơn GV cấp mầm non 0,5 lần (IC95\%: 0,35-0,72, $p<0,001$ ), kết quả này cũng phần nào được giải thích thông qua kết quả của mối liên quan giữa trình độ học vấn và tỷ lệ chấp nhận vắc xin, trình độ sau đại học chấp nhận ít hơn 0,43 lần (IC95\%:0,21 $0,85, p<0,05)$ so với trình độ trung cấp, kết quả này ngược với kết quả nghiên cứu tại Mỹ cũng như nhiều nước trên thế giới (Những người có trình độ THCS hoặc không có trình độ học vấn hay trình độ học vấn cơ bản có nhiều khả năng không dùng vắc xin COVID-19 hơn so với người có trình độ $Đ H$ ) [5] [6]. Tình trạng mắc bệnh mạn tính làm giảm quyết định chấp nhận tiêm vắc xin so với nhóm không mắc $\mathrm{OR}=0,48$ (IC95\%0,35 - 0,66, p<0,001), kết quả tương ứng với nghiên cứu của Bono và cộng sự (2021) (aOR: $0.81,95 \%$ CI $[0.71,0.92], \mathrm{p}=0.001$ ) (9). Điều đó cho thấy những lo lắng về bệnh mạn tính có thể sẽ ảnh hưởng đến các phản ứng của vắc xin và làm tăng tỷ lệ từ chối vắc xin ở nhóm đối tượng này, trong khi đây lại là đối tượng ưu tiên trong các chiến dịch tiêm vắc xin phòng COVID-19.

\section{KẾT LUẬN}

Tỷ lệ chấp nhận tiêm vắc xin phòng COVID19 trên 1902 là giáo viên các cấp từ mầm non đến THPT cho thấy tỷ lệ chấp nhận tiêm vắc xin chiếm tỷ lệ cao $(85,9)$. Các yếu tố liên quan có ý nghĩa thống kê là nhóm tuổi từ 40 đến 49 và 50 đến 59 cao hơn nhóm tuổi từ 18 đến 29 , tỷ lệ giáo viên mầm non sẵn sàng tiêm vắc xin cao hơn nhóm giáo viên THCS và THPT. ĐTNC là nhóm có gia đình tỷ lệ chấp nhận tiêm cao hơn so với nhóm còn độc thân và nhóm mắc bênh mạn tính làm giảm khả năng chấp nhận tiêm vắc xin so với nhóm không mắc bệnh.

\section{TÀI LIÊU THAM KHẢO}

1. https://covid19.who.int/

2. https://ncov.moh.gov.vn/

3. El-Elimat $\mathbf{T}$, AbuAlSamen MM, Almomani BA, Al-Sawalha NA, Alali FQ. Acceptance and attitudes toward COVID-19 vaccines: A crosssectional study from Jordan. PLoS One. $2021 \mathrm{Apr}$ 23;16(4):e0250555.

doi:10.1371/journal.pone.0250555.PMID:3389166 0;PMCID:PMC8064595.

4. Alqudeimat $Y$, Alenezi $D$, AlHajri B, Alfouzan H, Almokhaizeem Z, Altamimi S, Almansouri W, Alzalzalah S, Ziyab AH. Acceptance of a COVID-19 Vaccine and Its Related Determinants among the General Adult Population in Kuwait. Med Princ Pract. 2021;30(3):262-271. doi: 10.1159/000514636. Epub 2021 Jan 22. PMID: 33486492; PMCID: PMC8089409.

5. Al-Qerem WA, Jarab AS. COVID-19 Vaccination Acceptance and Its Associated Factors Among a Middle Eastern Population. Front Public Health. 
2021 Feb 10;9:632914. doi: 10.3389/ fpubh.2021.632914. PMID: 33643995; PMCID: PMC7902782.

6. Malik AA, McFadden SM, Elharake J, Omer SB. Determinants of COVID-19 vaccine acceptance in the US. EClinicalMedicine. 2020 Sep;26:100495. doi: 10.1016/j.eclinm.2020.100495. Epub 2020 Aug 12. PMID: 32838242; PMCID: PMC7423333.

7. Wang J, Jing R, Lai $X$, Zhang $H$, Lyu $Y$, Knoll MD, Fang H. Acceptance of COVID-19 Vaccination during the COVID-19 Pandemic in China. Vaccines (Basel). 2020 Aug 27;8(3):482. doi: 10.3390/vaccines8030482. PMID:

32867224; PMCID: PMC7565574.

8. Yuan Liang Woon and al. Factors influencing acceptance of COVID-19 vaccination among Malaysian adults, August 2020, National Conference for Clinical Research DOI: $10.13140 /$ RG.2.2.32024.62728

9. Bono, Suzanna Awang et al. "Factors Affecting COVID-19 Vaccine Acceptance: An International Survey among Low- and Middle-Income Countries." Vaccines vol. 9,5 515. 17 May. 2021, doi:10.3390/vaccines 9050515

\section{PHÂN TÍCH MộT SỐ YẾU TỐ LIÊN QUAN ĐẾN TỬ VONG Ở BỆNH NHÂN NHỒI MÁU NÃO CẤP TÍNH DIỆN RộNG CÓ ĐẠTT NộI KHÍ QUẢN}

\section{TÓM TẮT}

Mục tiêu: Phân tích một số yếu tố liên quan đến tử vong ở bênh nhân nhồi máu não cấp tính diên rông có đăt nội khí quản (NKQ). Đối tượng nghiên cứu: 69 bệnh nhân nhồi máu não cấp tính diện rộng, trong đó 38 bệnh nhân không đặt NKQ, 31 bệnh nhân có đăt NKO điều tri tai Trung tâmthần kinh Bênh viên Bạch Mai từ tháng 8/2020-7/2021. Phương pháp nghiên cứu: Mô tả cắt ngang. Kết quả: Tuổi trung bình của nhóm bênh nhân có đă̆t NKQ là $70,32 \pm 11,37$, nam giới chiếm $67,7 \%$, tỷ lề tử vong là $41,9 \%$, điểm NIHSS trung bình khi nhập viện là $21,81 \pm 3,53$, điểm Glasgow lúc nhâp viên là $11,87 \pm 1,15$. Các triệu chứng lâm sàng khi khởi phát nổi bât hơn ở nhóm có đă̆t NKQ bao gồm: đau đâu $(48,1 \%)$, rối loạn ý thức $(90,3 \%)$, quay mắt quay đầu $(77,4 \%)$, rối loạn cơ tròn $(74,2 \%)$, với $p<0,05$. Lý do đặt nội khí quản do nguyên nhân thần kinh chiếm 80,6\%; điểm Glasgow trung bình khi đặt nôi khí quản

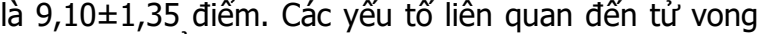
bao gồm: điểm NIHSS lúc nhập viện, đặt nội khí quản do nguyên nhân thần kinh, điểm Glasgow lúc đặt nội khí quản, bất thường kích thước đồng tử và mất PXAS đồng tử lúc đặt nội khí quản, mức độ di lệch đường giữa trên hình ảnh học. Kết luận: Tỷ lệ tử vong ở nhóm bênh nhân nhồi máu não cấp tính diện rộng có đặt nội khí quản là 41,9\%. Các yểu tố liên quan đến tử vong bao gồm: điểm NIHSS lúc nhâp viên, đăt nôi khí quản do nguyên nhân thần kinh, điểm Glasgow lúc đătt nội khí quản, bất thường kích thước đồng tử và mất PXAS đồng tử lúc đặt nội khí quản, mức độ di lệch đường giữa trên hình ảnh học. Tư khóa: Nhồi máu não diện rộng, nội khí quản, tử vong.

${ }^{1}$ Bệnh Viện Đa khoa Của Đông - Nghệ An

2Trường đại họ Y Hà Nội

${ }^{3}$ Bênh viên Bach Mai

Chịu trách nhiệm chính: Nguyễn Văn Quân

Email: bsquantk@gmail.com

Ngày nhận bài: 17.5.2021

Ngày phản biên khoa hoc: 2.7.2021

Ngày duyệt bài: 19.7.2021
Nguyễn Văn Quân ${ }^{1}$, Võ Hồng Khôi ${ }^{2,3}$

SUMMARY

ANALYSIS OF SOME FACTORS RELATED TO

MORTALITY IN PATIENTS WITH ACUTE

LARGE HEMISPHERIC INFARCTION WITH

ENDOTRACHEAL INTUBATION

Background: Analysis of some factors related to mortality in patients with acute large hemispheric infaection with endotracheal intubation. Method: Study of69 patients with acute large hemispheric infaection, of which 38 patients without the intubation and 31 patients with the intubation, were treated in Neurological Center of Bach Mai Hospital from August 2020 to July 2021. Result: The average age of patients with the intubation was $70.32 \pm 11.37$, male $69.2 \%$, mortality rate $41,9 \%$. The mean of NIHSS score at hospital admission was $21.81 \pm 3.5$. The mean of Glasgow score at hospital admission was $11.87 \pm 1.15$. Clinical symptomonset was more prominent in the group with the intubation including: headache $(48.1 \%)$, consciousness disorders $(90.3 \%)$, head-eye deviation (77.4\%), urinary incontinenceonset $(74.2 \%)$, with $p<0.05$. Reason for the intubation due to neurological causes accounted for $80.6 \%$; The mean Glasgow score at intubation was $9.10 \pm 1.35$ points. Factors related to the mortality included: NIHSS score at hospital admission, endotracheal intubation due to neurological causes, Glasgow score at the intubation, dilated pupi admission and loss of light reflection at the intubation, midline shift. Conclusion: The mortality rate in the group of patients with acute large hemispheric infaection with the intubation was $41.9 \%$. Factors related to the mortality included: NIHSS score on admission, neurogenic reason for intubation, Glasgow score at intubation, dilated pupil admission and loss of light reflection at the intubation, midline shift

Keywords; Acute large hemispheric infaection, endotracheal intubation, morality.

\section{I. ĐĂT VẤN ĐỀ}

Đột quy não là vấn đề thời sự cấp thiết trên toàn câu, là nguyên nhân tử vong đứng hàng 